Review Article

\title{
Gene Editing Nucleases - ZFNs, TALENS and CRISPR
}

Shiva Krishna $G^{*}$, Suma $K^{* *}$

${ }^{*}$ Clinical Embryologist and Cell Biologist, ${ }^{* *}$ Gynaecologist, Managing Director, Prasad Research Foundation, Secunderabad, Andhra Pradesh, India.

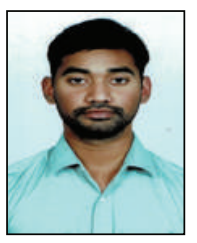

Dr.Shiva Krishna is working as Consultant Embryologist and Cell Biologist in Prasad Research Foundation. He completed his undergraduation from Army College of Dental Sciences and did his Post Graduate Diploma in Clinical Embryology from the Dept. of Andrology and Reproductive Medicine, Chettinad Hospital and Research Institute. He is currently pursuing his Ph.D from OPJS University.

Corresponding author - Dr. Suma Kantipudi - (info@prasadresearch.org)

Chettinad Health City Medical Journal 2019; 8(4): 130 - 135

DOI: https://doi.org/10.36503/chcmj 8(4)-06

\begin{abstract}
Meganucleases can induce local mutagenesis and intentional Double Strand Break (DSB) but the target specificity is low with the Meganucleases. Experiments with Type IIS Restriction enzymes showed that it cannot be useful as they typically recognise 4-8bp palindromic DNA and on average cut DNA once every 4016-65, 536 bases. In addition, there is an overlap of cleavage domain and endonuclease domain in the Type lls Restriction enzyme. On Further Research, Zinc Finger Nucleases (ZFNs), Transcription Activator Like Effector Nucleases (TALENs) and CRISPR CAS 9 has been discovered and considered as advanced engineering Nucleases due to its target specificity and efficiency. This review generally discusses the advanced engineering nucleases in terms of the structure, mechanisms of action,specificity and target efficiency.
\end{abstract}

Key words : Gene editing, Nucleases, DNA, Zinc finger Nucleases, TALENS, CRISPR.

\section{Introduction}

The making of specific changes in DNA with the help of technology is known as Gene editing. Rare-cutting Meganucleases were used earlier for gene editing but the target specifity is low with Homing Endonuclease. ${ }^{1}$ On Further Research, three advanced engineering nucleases has been discovered. They are Zinc Finger Nucleases (ZFNs), TALENs (Transcription Activator Like Effector Nucleases), and CRISPR CAS9. ${ }^{2}$

\section{History Of ZFNs, TALENS And CRISPR}

Klug and co-workers identified zinc motifs in Xenopus oocytes in 1985. ${ }^{3}$ The crystal structure of the zif-268 complex was studied by Pavetich and Pabo in 1991 who gave a clear idea of how ZFNs recognise their cognate sites. ${ }^{4}$ TYPE II (R-M) Restriction endonuclease acts as a cleavage domain for ZFNs and TALENs.4 The fusion of DNA binding Domain to the target site of DNA will send signals to Cleavage domain through allosteric reactions. ${ }^{1}$ Kim and Chandrasegaran proposed the first chimeric endonuclease by ubx fusion to Fokl cleavage domain in $1994 .^{5}$ ZFN's was first used in human cells in 2003 by Porteus and Baltimore.1 TALE motifs was discovered in plant virulence factors from Xanthomonas bacteria in 2009. ${ }^{6}$ TALENs was proposed by Christian et al in 2010 by fusing TALE motifs with endonuclease domain. ${ }^{7}$ CRISPR was discovered in 1987 but its importance in genome engineering started with the proposal of Cas9-crRNA complex as RNA-guided endonuclease in 2012. ${ }^{1-8}$

\section{Zinc Finger Nucleases}

Zinc Finger Nucleases encompasses DNA binding Domain and endonuclease Domain. ${ }^{9}$ DNA binding Domain is an array of site-specific domains that ranges its length from 9-18 bp based on the target specificity9 (Fig 1). Binding Domain attaches to the Endonuclease domain which comprise of the endonuclease domain of the bacterial Fokl restriction enzyme. ${ }^{2-9} \mathrm{~A}$ pair of Zinc finger nucleases are designed to target a specific site in which one will flank on the forward strand and others will overlap on the respective complimentary strand. Fok1 restriction domain pair dimerize on reaching either side of DNA strands cleave the target with a double-strand break with 51 overhangs. ${ }^{9}$ Cells repair Double-strand breaks by two ways; 1) Non-Homologous End Joining and 2) Homology Directed Repair. Non Homologous End Joining (NHEJ) can occur at any phase of the cycle and occasionally it might result in fallacious repair.10 The fallacious repair of NHEJ can be utilised to introduce frameshifts into the coding sequence of a gene, potentially knocking out the gene by a combination of two mechanisms: premature truncation of the protein and nonsense-mediated decay of the mRNA transcript, the latter of which is not always particularly efficient. ${ }^{10}$ Alternatively, HDR can be 
deployed to insert a particular mutation, with the insertion of a repair template containing the desired mutation flanked by homology arms. Homology Directed Repair occurs during late s phase or $\mathrm{G}_{2}$ phase when the sister chromatids are available as a repair template. ${ }^{10}$

Even though ZFNs acts as a promising tool for gene editing, it has some disadvantages. ZFNs has not proven straight forward to bind an extended stretch of nucleotides with high affinity." To vanquish this difference, an academic consortium has developed about the protocol and selection of zinc finger components to obtain the desired sequence with high affinity. ${ }^{12,13}$ Nevertheless, it will take many months for Nonexperts to get the desired sequence with high affinity. 12,13 Another potential disadvantage is that target site selection is limited, the open-source ZFNs constituents can only be used to target binding sites every 200 bps in random DNA sequence (of note, commercial sources of ZFNs offer higher design densities, with the possibility to target binding sites every 50 bps in random DNA sequences). ${ }^{14-17}$ While this may be a non-issue if an investigator seeks to knock out a gene, since a frameshift introduced anywhere in the early coding sequence of the gene can produce the desired result, it may present challenges if a specific site is required, e.g., to knock in a specific mutation. ${ }^{14-17}$ Since the introduction of the open-source platform, alternative platforms to engineer optimized ZFNs have emerged, each with varying degrees of speed, flexibility in site selection, and success rates. The concern about the off-target sites is associated with ZFNs and other gene-editing nucleases. In a study on Human pluripotent stem cells, 10 probable off-target sites were estimated based on its high-frequency similarity with the on-target site. Nonetheless, one off-target mutation was detected out of 184 clones. $^{18}$ Later, two studies of ZFNs seeking to identify potential off-target sites for several ZFN pairs revealed off-target events at numerous loci in a cultured human tumour cell line. Thus, scientists should be perspicacious of the possibility that ZFNs designed for a particular purpose may incur undesired off-target events at a low rate. The action of ZFNs can be reduced on off-target sites by using use a pair of ZFNs that have distinct Fokl domains that are obligate heterodimers and Introduction of purified ZFNs into cells. ${ }^{19-22}$

Zinc-finger nucleases (ZFNs)

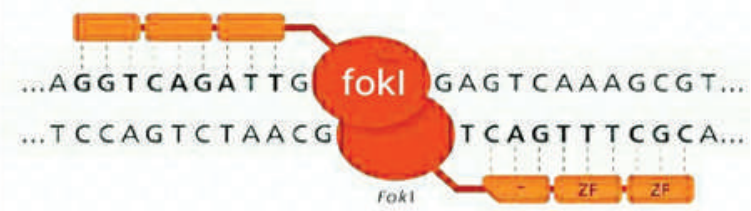

Figure 1: Zinc Finger Nucleases with Fok1 Domains and zinc fingers occupying 9 base pairs

\section{TALENs}

Transcription Activator Like Effector Nucleases (TALENs) has been introduced in the year 2009 while studying xanthomonas bacteria, which are rather common pathogen affecting multiple plant species. ${ }^{23}$ TALENs contains TAL effectors and endonuclease Domain which is structurally similar to ZFNs. The naturally occurring TALE repeats contains tandem arrays with 10 to 30 repeats that bind and recognize extended DNA sequence. ${ }^{24}$ Each repeat is 33-35 amino acids with site specific 12 and 13th position which is known as Repeat Variable Diresidues (RVD). Each RVD specify the identity of a single base thus making the tandem array of repeats and helps in specific recruitment of the DNA molecule(fig-2). ${ }^{25-29}$ The "code-like" relationship between amino acid sequence of RVD and recognized/bound nucleotides allows for the engineering of specific DNA-binding domains in a manner very much appropriate to the assembly of structures with Lego bricks. RVD mediates specific nucleotide recognition of TALE that a two amino acids repeat precisely recognizes and binds one base pair. The following recognition preferences have been experimentally identified: $\mathrm{HD}=\mathrm{C} ; \mathrm{NG}=\mathrm{T} ; \mathrm{NI}=$ $\mathrm{A} ; \mathrm{NS}=\mathrm{A}, \mathrm{C}, \mathrm{G}$, or $\mathrm{T} ; \mathrm{NN}=\mathrm{A}$ or $\mathrm{G} ;$ and $\mathrm{IG}=\mathrm{T} .{ }^{30}$

TALENs have some advantages and disadvantages when compared to ZFNs. ZFNs require 9-18 bp to undergo gene editing where TALE repeat array can be easily extended to whatever length is desired.TALENs are often built to $18 \mathrm{bp}$ or more, however recent evidence suggest longer length may lead to less specifity.31 TALENs need fewer constraints over ZFNs on site selection, in theory, there are multiple possible TALENs pairs available for each bp of a random DNA sequence. ${ }^{32}$ TALENs express at off-target sites which are a concern even with ZFNs.A study conducted on Human induced pluripotent stem cells where TALENs were used for gene editing,19 off target sites were detected based on its similarity to the on-target sites. ${ }^{33}$ Although data is scarce, in a study conducted on CCR5 gene,TALENs and ZFNs were used for gene editing. TALENs produced fewer off-target mutations than ZFNs at a highly similar site in CCR2 gene. ${ }^{34}$ To reduce the off-target mutations obligate heterodimers of ZFNs and TALENs. Recently, whole-genome sequencing studies of human pluripotent stem cell clones edited with improved TALENs showed that the overall off-target event rate is very low. ${ }^{35,36}$

The disadvantage associated with TALENs is its large size. The size of cDNA coding for TALENs is $3 \mathrm{~kb}$ and the size of cDNA coding for ZFNs is $1 \mathrm{~kb}$. This larger size makes it harder to use for the therapeutic applications as it is harder to deliver TALENs into the cells when compared to ZFNs due to its smaller size. The Viral Vectors (such as adeno associated virus [AAV], with less than $5 \mathrm{~kb}$ ) or as RNA molecules are 


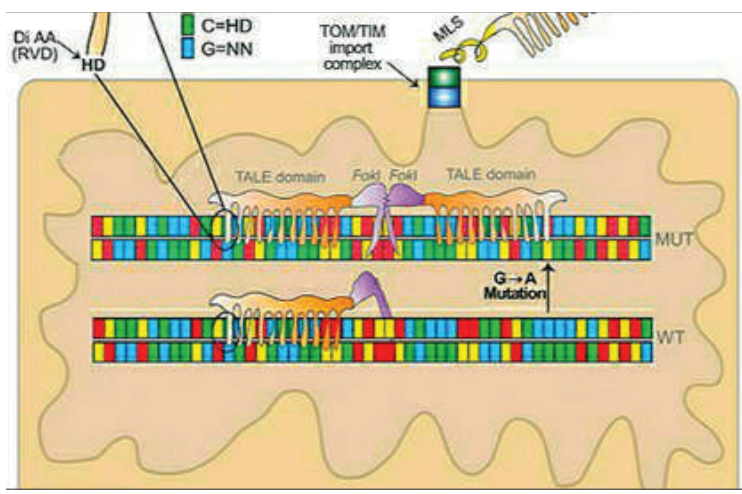

Figure 2: TALE domain with fok1 and specific RVD region at 12 and 13 th position

used for delivery will keep TALENs in an inappropriate situation to use. Furthermore, the highly repetitive nature of the TALENs may weaken their ability to be packaged and delivered by some viral vectors.This situation can apparently be overcome by permutating the coding sequences of the tale repeats. 37,38

\section{CRISPR}

CRISPR stands for Clustered Regularly Interspaced Short Palindromic Repeats which is bacterial and archaeal adaptive immune system. 39 The past invaders enter the bacteria and integrate with the DNA sequence. Acquired sequences allow bacteria to identify the viral or plasmid invaders and deletes it from integrating into DNA sequence. ${ }^{40,41}$

CRISPR immunity has distinct phases. During the adaptive phase, bacteria acquires the memory. Scientists investigated the acquired memory and discovered a highly variable region in the bacteria known as CRISPR locus. The distinct feature is many non-contiguous repeats separated by variable region termed spacers. ${ }^{42}$ Upon close investigation, the spacer region is identical to phase resistant bacteria. Thus, spacer region correlated with phage resistance leading to the model that short regions of the invader's genome are integrated into the CRISPR loci as a spacer, separated by repeat sequences, resulting in the cellular memory of the previous infections. 43 Three types of CRISPR system exist. They are Type1,Typez and Type3. CRISPR system 2 is most commonly used for genome editing due to its simplicity. ${ }^{44}$ The immune system of Bacteria generates CRISPR RNA(cr RNA) from CRISPR locus which is known as Pre CRNA. A second RNA is transcribed upstream of CRISPR locus which is known as transactivating RNA(tracr RNA). Tracr RNA is complimentary to repeat of CRISPR locus and binds to the newly transcribed pre-crRNA creating a double-stranded RNA that gets cleaved by RNaselll (an enzyme that recognizes and cuts double-stranded RNA) resulting in a crRNA:tracrRNA complex containing just one spacer sequence. Once casg binds to the RNA complex, it acts like endonuclease and cleaves DNA sequence complimentary to $\mathrm{cr}$ RNA. ${ }^{45}$ Searching the invading DNA for sequences complementary to the crRNA takes place with Casg binding to sequences in the invading viral or plasmid genome termed Proto-spacer Adjacent Motifs or PAMs.46,47 Four different research groups; Mali et al, Wright et al, Jinek et al, Swiech et al started working on an adaptation of prokaryotic cell adaptive immune system for gene editing.CRISPR system which is used for therapeutic purposes contains single guided RNA (sgRNA), Cas9 which acts like a helicase, endonuclease for unwinding and cut the target DNA and PAM sequence which helps in identifying the Target DNA and reduce off-targets (Fig3). Different bacteria has different PAM sites. ${ }^{47}$ The 51-NGG-31 PAM which is commonly used for gene editing is obtained from S.pyogenes (spcas9). Arg1333 and Arg1335 are two critical arginine residues in SpCasg that interact with the guanine nucleases of the PAM on the noncomplementary strand. ${ }^{48}$ This interaction between the guanines of the PAM and the arginines in SpCasg positions the phosphate of the DNA backbone 5' to the PAM to interact with a phosphate-lock loop in Cas9 and helps in DNA strand unwinding. If the DNA is complementary to the guide RNA, an RNA: DNA hybrid forms, called an R loop, and DNA cleavage results from the action of two different Casg nuclease domains: the HNH domain nicks the DNA strand that is complementary to the crRNA and the RuvC-like domain nicks the non-complementary strand to the crRNA. Casg cleaves the DNA 3 base pairs upstream of the PAM and results in a blunt-end cleavage of DNA. Cleaving the DNA is deleterious to the invading plasmid or virus and leads to degradation and protection against these invaders. ${ }^{44}$ In type II CRISPR systems, the PAM-proximal 10-12 nucleotides located in the 3 ends of the 20-nt spacer sequence is termed as the seed region. Mismatches in this seed region severely impair or completely revoke target DNA binding and cleavage. 49

Nickases are introduced in CRISPR which serves as a better option in homologus end repair. Nickases will only break one strand .D10 mutation will inactivate the RuvCdomain and only the target strand is cleaved where H840A will inactivate the $\mathrm{HNH}$ domain and Nontarget strand undergoes cleavage. 49 The orientation of guided RNA place a crucial rule when Nickases targeting at different strands to create a DSB break. Base editing was developed by Liu and co-workers developed by combining three proteins: a cytidine deaminase, a natural enzyme that converts $C$ to uridine (U); a mutated Cas9 CRISPR enzyme that doesn't break DNA but uses an associated guide RNA to target specific DNA sequences; and a protein that prevents reversion of $\mathrm{U}$ back to $\mathrm{C} .{ }^{\circ}$ After the cytidine deaminase changes 


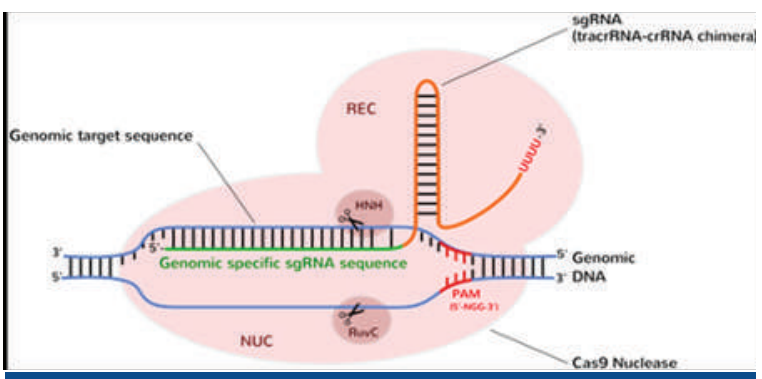

Figure 3: : Casg Nuclease domain with REC and NUC lobe. SgRNA is a target sequence to be cleaved and PAM sequence which helps in identifying target DNA

$\mathrm{C}$ to $\mathrm{U}$, the base editor nicks the nontarget strand to induce cellular machinery to replace $G$ with $A$ and change $U$ to $T$. Changing AT to $G C$ is a much more difficult task than going from CG to TA because no natural enzyme converts $A$ to a base resembling $G$ or changes $T$ to a C-like base in DNA. Liu and co-workers overcome the problem by using directed evolution and enzyme engineering to convert a bacterial adenosine deaminase that normally works in RNA into a deoxyadenosine deaminase that converts $A$ to inosine (I) in DNA. The researchers use a conjugate of the deoxyadenosine deaminase with a catalytically impaired Casg to convert A to I at a target site and to nick the opposite strand. The cell's machinery then completes the process, converting I to $\mathrm{G}$ and $\mathrm{T}$ to $\mathrm{C} .{ }^{50}$
One of the disadvantages with CRISPR is its huge size.51, 52 The cDNA encoding $\mathrm{S}$. pyogenes Cas9 (Spcas9) is approximately $4.2 \mathrm{~kb}$ in size, which is bigger than TALEN monomer and ZFN monomer (though both TALENs and ZFNs require dimerization, making their effective sizes larger).52Larger size of CRISPR CAS9 along with single guided RNA cannot be accommodated into the vectors.SpCas9 will recognise only NGG pam site which demands a need of different Cas9 variants to recognise the PAM site.52 Off-target sites are also a concern and can be reduced with Casg variants. ${ }^{52}$

A few Phase 1 Clinical trials have been done with ZFNs,TALENs and CRISPR-CAS9. 53 The earliest clinical trial was done with ZFNs in HIV patients.ZFNs were used to knock out the CCR5 coreceptor gene in HIV positive patients there by making $T$ cells resistant to virus.53 TALENs have been used to enhance the efficacy of CAR-T cells and two trials have been done with CRISPR CAS9 to enhance the efficacy of CAR-T cells. 53

\section{Summary}

ZFNs, TALENs and CRISPR serves as gene-editing tools.TALENs are considered to be better than ZFNs due to its bigger binding domain which helps in minimal off-target sites.ZFNs and TALENs are methylation sensitive which leads to DNA methylation

\begin{tabular}{|c|c|c|c|c|}
\hline $\begin{array}{c}\text { Serial } \\
\text { No. }\end{array}$ & Parameter & ZFNs & TALENS & CRISPR/Cas9 \\
\hline 1 & Design simplicity & $\begin{array}{l}\text { Moderate(ZFNs } \\
\text { need customized } \\
\text { protein for every } \\
\text { DNA sequence) }\end{array}$ & $\begin{array}{l}\text { Slightly complex } \\
\text { (identical repeats are } \\
\text { multiple, which } \\
\text { creates technical } \\
\text { issues of engineering } \\
\text { and delivery into the } \\
\text { cell) }\end{array}$ & $\begin{array}{l}\text { Simpler(available } \\
\text { versions for crRNA can } \\
\text { be easily designed) }\end{array}$ \\
\hline 2 & $\begin{array}{l}\text { Engineering } \\
\text { feasibility }\end{array}$ & Low & Higher & Highest \\
\hline 3 & $\begin{array}{l}\text { Large scale library } \\
\text { preparation }\end{array}$ & $\begin{array}{l}\text { Not much } \\
\text { progress(individual } \\
\text { gene tailoring } \\
\text { required) }\end{array}$ & $\begin{array}{l}\text { Not much progress( } \\
\text { individual gene } \\
\text { tailoring required) }\end{array}$ & $\begin{array}{l}\text { The progress } \\
\text { demonstrated(CRISPR } \\
\text { only requires plasmid } \\
\text { containing small } \\
\text { oligonucleotides ) }\end{array}$ \\
\hline 4 & $\begin{array}{l}\text { Multiplex genome } \\
\text { editing }\end{array}$ & Few models & Few models & $\begin{array}{l}\text { High yielding } \\
\text { multiplexing available }\end{array}$ \\
\hline 5 & Specificity & Low & Higher & $\begin{array}{l}\text { Low(may be increased } \\
\text { due to Nickase) }\end{array}$ \\
\hline 6 & Efficiency & Normal & Normal & High \\
\hline 7 & Cost & Low & High & Low \\
\hline 8 & $\begin{array}{l}\text { Mutagenic } \\
\text { throughput }\end{array}$ & Low & Moderate & High \\
\hline
\end{tabular}


and decrease in editing efficiency comparable to CRISPR which is not methylation-sensitive.CRISPR is a cost-effective and non-laborious process due to its availability in nature. CRISPR design is simple, cost-effective and can undergo multiplex genome editing. DNA Recognition is simple by forming a Ribonucleotide complex with CRISPR. ZFNs and TALENs undergoes protein/DNA recognition process which is bothersome.zinc motifs design in ZFNs is a laborious process the design need to bechecked in the Library and the integrity of each zinc motifs is important for design and it has low specificity due to its smaller structure.TALENs has got bigger size which increases the specificity but cannot be incorporated into the vectors due to its large size. CRISPR is simple because of single unit structure and it is available in nature and shows multiplex genome editing,high specificity and low cost for synthesis has made many researchers attract towards CRISPR. However, all three has got off-target sites. All three nucleases need further clinical trials to be used for therapeutic use.

\section{References}

1. Chandrasegaran S, Carroll D . Origins of Programmable Nucleases for Genome Engineering. J Mol Biol. 2016;428(5):963-89.

2) Gupta RM, Musunuru K. Expanding the genetic editing tool kit: ZFNs, TALENs, and CRISPR-Cas9. J Clin Invest. 2014;124(10):4154-61.

3) J Miller, A D McLachlan, A. Klug. Repetitive zinc-binding domains in the protein transcription factor IIIA from Xenopus oocytes. EMBO J.1985;4(6):1609-14.

4) Pavletich NP, Pabo CO Zinc finger-DNA recognition: crystal structure of a Zif268-DNA complex at 2.1 A. Sci.1991;252(5007):809-17.

5) Y G Kim, S Chandrasegaran. Chimeric restriction endonuclease. Proc Natl Acad Sci U S A. 1994;91(3):883-7.

6) Moscou MJ, Bogdanove AJ. A simple cipher governs DNA recognition by TAL effectors.Sci. 2009;326(5959):1501.

7) MichelleChristian. Targeting DNA Double-Strand Breaks with TAL Effector Nucleases.Genetics. 2010;186(2):757-761.

8) G Gasiunas. Cas9-crRNA ribonucleoprotein complex mediates specific DNA cleavage for adaptive immunity in bacteria. Proc Natl Acad Sci USA. 2012;109(39):2579-86.

9) Urnov FD, Rebar EJ, Holmes MC, Zhang HS, Gregory PD. Genome editing with engineered zinc finger nucleases. Nat Rev Genet. 2010;11(9):636-646.
10) Rouet P, Smih F, Jasin M. Expression of a site specific endonuclease stimulates homologous recombination in mammalian cells. Proc Natl Acad Sci USA. 1994;91(13):6064-8.

11) Ramirez CL. Unexpected failure rates for modular assembly of engineered zinc fingers. Nat Methods. 2008;5(5):374-5.

12) Maeder ML. Rapid "open-source" engineering of customized zinc-finger nucleases for highly efficient gene modification. Mol Cell. 2008;31(2):294-301.

13) Maeder ML, Thibodeau-Beganny S, Sander JD, Voytas DF, Joung JK. Oligomerized pool engineering (OPEN): an 'open-source' protocol for making customized zinc-finger arrays. Nat Protoc. 2009;4(10):1471-501.

14) Sander JD. Selection-free zinc-finger-nuclease engineering by context-dependent assembly (CoDA). Nat Methods. 2011;8(1):67-69.

15) Gupta A, Christensen RG, Rayla AL, Lakshmanan A, Stormo GD, Wolfe SA. An optimized two finger archive for ZFN-mediated gene targeting. Nat Methods. 2012;9(6):588-90.

16) Bhakta MS. Highly active zinc-finger nucleases by extended modular assembly. Genome Res. 2013;23(3):530-8

17) Gaj T, Guo J, Kato Y, Sirk SJ, Barbas CF. Targeted gene knockout by direct delivery of zinc-finger nuclease proteins. Nat Methods. 2012; 9(8):805-7.

18) Kim HJ, Lee HJ, Kim H, Cho SW, Kim JS. Targeted genome editing in human cells with zinc finger nucleases constructed via modular assembly. Genome Res. 2009;19(7):1279-88.

19) Hockemeyer D. Efficient targeting of expressed and silent genes in human ESCs and iPSCs using zinc-finger nucleases. Nat Biotechnol. 2009;27(9):851-7.

20) Gaj T, Guo J, Kato Y, Sirk SJ, Barbas CF. Targeted gene knockout by direct delivery of zinc-finger nuclease proteins. Nat Methods. 2012;9(8):805-807.

21) Doyon Y. Enhancing zinc-finger-nuclease activity with improved obligate heterodimeric architectures. Nat Methods. 2011;8(1):74-9.

22) Szczepek M, Brondani V, Buchel J, Serrano L, Segal DJ, Cathomen T. Structure-based redesign of the dimerization interface reduces the toxicity of zinc-finger nucleases. Nat Biotechnol.2007; 25(7):786-93.

23) Miller JC. An improved zinc-finger nuclease architecture for highly specific genome editing. Nat Biotechnol. 2007;25(7):778-85

24) J. Keith Joung ,Jeffry D. Sander.TALENs: a widely applicable technology for targeted genome editing. Nat Rev Mol Cell Biol. 2013;14(1): 49-55. 
25) Bogdanove AJ, Voytas DF. TAL effectors: customizable proteins for DNA targeting. Science. 2011;333(6051):1843-6.

26) Moscou MJ, Bogdanove AJ. A simple cipher governs DNA recognition by TAL effectors. Science. 2009;326(5959):1501.

27) Boch J. Breaking the code of DNA binding specificity of TAL-type III effectors. Science. 2009; 326(5959):1509-12.

28) Morbitzer R, Romer P, Boch J, Lahaye T. Regulation of selected genome loci using de novo engineered transcription activator-like effector (TALE)-type transcription factors. Proc Natl Acad Sci USA. 2010;107(50):21617-22.

29) Streubel J, Blucher C, Landgraf A, Boch J. TAL effector RVD specificities and efficiencies. Nat Biotechnol. 2012;30(7):593-5.

30) Cong L, Zhou R, Kuo YC, Cunniff M, Zhang F. Comprehensive interrogation of natural TALE DNA-binding modules and transcriptional repressor domains. Nat Commun.2012;3:968.

31) Radek Jankele,Petr Svoboda. TAL effectors: tools for DNA Targeting. Brief Funct Genomics. 2014;13(5):409-19.

32) Guilinger JP. Broad specificity profiling of TALENs results in engineered nucleases with improved DNA-cleavage specificity. Nat Methods. 2014;11(4):429-35.

33) Reyon D, Tsai SQ, Khayter C, Foden JA, Sander JD, Joung JK. FLASH assembly of TALENs for high-throughput genome editing. Nat Biotechnol. 2012;30(5):460-5.

34) Hockemeyer D. Genetic engineering of human pluripotent cells using TALE nucleases. Nat Biotechnol. 2011;29(8):731-4.

35) Mussolino C, Morbitzer R, Lutge F, Dannemann N, Lahaye T, Cathomen T. A novel TALE nuclease scaffold enables high genome editing activity in combination with low toxicity. Nucleic Acids Res. 2011;39(21):9283-93.

36) Smith C. Whole-genome sequencing analysis reveals high specificity of CRISPR/Cas9 and TALEN-based genome editing in human iPSCs. Cell Stem Cell. 2014;15(1):12-13.

37) Suzuki K. Targeted gene correction minimally impacts whole-genome mutational load in human-disease-specific induced pluripotent stem cell clones. Cell Stem Cell. 2014;15(1):31-6.

38) Holkers M. Differential integrity of TALE nuclease genes following adenoviral and lentiviral vector gene transfer into human cells. Nucleic Acids Res. 2013;41(5):63.

39) Yang L, et al. Optimization of scarless human stem cell genome editing. Nucleic Acids Res. 2013;41(19):9049-61.
40) Avner Hershlag, Sara L. Bristow.Editing the human genome: where ART and science intersect.JAss Rep Gen.2018;35:1367-70.

41) Van Der Oost J, Jore MM, Westra ER, Lundgren M. CRISPR-based adaptive and heritable immunity in prokaryotes. Trends Biochem Sci. 2009;34(8):401-7.

42) Horvath P, Barrangou R. CRISPR/Cas, the immune system of bacteria and archaea. Science. 2010;327(5962):167-170.

43) Bolotin A, Quinquis B, Sorokin A, Dusko S. Ehrlich clustered regularly interspaced short palindrome repeats (CRISPRs) have spacers of extrachromosomal origin. Microbiology.2005;151: 2551-61.

44) Barrangou R, Fremaux C, Deveau H, Richards $M$, Boyaval P, Moineau S, et al. CRISPR provides acquired resistance against viruses in prokaryotes. Science.2007;315:1709-12.

45) Fuguo Jiang,Jennifer A Doudna.CRISPR-Cas9 Structures and Mechanisms. Annu. Rev. Biophys. 2017;46:505-29.

46) Deltcheva E, Chylinski K, Sharma CM, Gonzales K, Chao Y, Pirzada Z A, et al.CRISPR RNA maturation by trans-encoded small RNA and host factor RNase III. Nature. 2011;471:602-7.

47) Sternberg SH, Redding S, Jinek M, Greene EC, Doudna JA.DNA interrogation by the CRISPR RNA-guided endonuclease Casg. Nature. 2009;507:62-7.

48) Gasiunas G, Barrangou R, Horvath P, Siksnys V. Cas9-crRNA ribonucleoprotein complex mediates specific DNA cleavage for adaptive immunity in bacteria. Proc. Natl. Acad. Sci. USA.2012;109:2579-86.

49) Anders C, NiewoehnerO, Duerst A, Jinek $M$. Structural basis of PAM-dependent target DNA recognition by the Cas9 endonuclease. Nature. 2012;513:569-73.

50) Manuel Kaulich,Steven F Dowdy.Combining CRISPR/Casg and rAAV Templates for Efficient Gene Editing. Nucleic Acid Ther. 2015;25(6):287-96.

51) Holly A, Rees David R, Liu.Base editing: precision chemistry on the genome and transcriptome of living cells. Nat Rev Genet. 2018;19(12):770-88.

52) Omoyayi Ibrahim Omodamilola, Abdullahi Umar Ibrahim. CRISPR Technology.Advantages, Limitations and Future Direction. J Biomed Pharm Sci. 2018;1(2):115.

53) Hakim Manghwar, Keith Lindsey, Xianlong Zhang and ShuangxiaJin. CRISPR/Cas System: Recent Advances and Future Prospects for Genome Editing. Trends in Plant Science. 2019; 24(12):1102-25. 